real circulation, and the $\mathrm{LAD}$ was then incised for grafting. The MIDCAB doughnut was applied again around the LAD. Bothersome bleeding from the anastomotic site was easily controlled without aortic venting. After removal of the instrument, the traces on the heart surface disappeared in a few minutes. Hemorrhagic lesions was never found in the suction sites. The instrument was easy to handle when being placed over and removed from the heart surface.

The instrument has been applied in seven patients at other institutes during minimally invasive $\mathrm{CABG}$ without the support of extracorporeal circulation. In all seven patients, the LAD was bypassed with a left internal thoracic artery (LITA) bypass graft. Through a small left anterior thoracotomy incision, the MIDCAB doughnut was fitted over the anastomotic site of the LAD by air suction. Through this minimal access approach, stable immobilization of the LAD could also be achieved on the beating heart. The LAD was occluded proximally with a tourniquet and then incised with a knife. The distal LAD was not occluded with a looping suture; however, bothersome back bleeding from the distal coronary artery was almost completely controlled by this technique. The anastomotic site could be firmly stabilized, and the LITA-LAD anastomosis could be precisely completed, just as with cardioplegic arrest (Fig. 2). After completion of the anastomosis, negative pressure was released and the MIDCAB doughnut was cut off and removed from the LITA graft. Postoperative coronary angiography showed that all the grafts were widely patent, and there were no stenotic lesions at the suction sites. In two patients, percutaneous transluminal coronary angioplasty to the circumflex artery was performed simultaneously, All seven patients were discharged uneventfully from the hospital and are doing well. In conclusion, this technical procedure can stabilize the artery and control bleeding, making the anastomosis easier.

Comment. A new instrument called a MIDCAB doughnut has been developed for immobilization of the anastomotic site and control of bleeding during minimally inva- sive $\mathrm{CABG}$. The instrument can make the operative field both motionless and bloodless without snaring. The mechanism of the instrument is based on a negative pressure with fixation to the heart surface around the anastomotic site. Potential problems of encircling snares, such as septal artery injury, atheromatous embolism, coronary artery transection, ventricular tear, or late stenosis of the native coronary, are avoided by use of this instrument. Negative pressure of the instrument occludes the coronary vessels to reduce or interrupt bothersome bleeding from the proximal or distal coronary artery. The need for the proximal encircling suture to be pulled up firmly for immobilization is lessened. An Octopus device (Medtronic, Inc., Minneapolis, Minn.) reported by Borst and associates ${ }^{4}$ is based on a principle similar to ours; the device consists of hard multiple suction domes. In their study, light microscopy showed small hemorrhagic suction lesions and intact endothelium of the coronary vessels, which traversed suction lesions, with a negative pressure under $-400 \mathrm{~mm} \mathrm{Hg}$. We believe that negative pressure fixation of the instrument to the heart may be superior to local myocardial compression methods.

\section{REFERENCES}

1. Westaby S, Benetti FJ. Less invasive coronary surgery: consensus from the Oxford meeting. Ann Thorac Surg 1996;62: 924-31.

2. Subramanian VA, Sani G, Benetti FJ, et al. Minimally invasive coronary bypass surgery: a multi-center report of preliminary clinical experience. Circulation 1995;92(Suppl):I645.

3. Boonstra PW, Grandjean JG, Mariani MA. Improved method for direct coronary grafting without CPB via anterolateral small thoracotomy. Ann Thorac Surg 1997;63:567-9.

4. Borst C, Jansen EWL, Tullenken CA, et al. Coronary artery bypass grafting without cardiopulmonary bypass and without interruption of native coronary flow using a novel anastomosis site restraining device ("Octopus"). J Am Coll Cardiol 1996; 27:1356-64.

\title{
MINIMALLY INVASIVE REDO AORTIC VALVE REPLACEMENT
}

\author{
Robert K. W. Tam, FRACS, R. Bruce Garlick, FRACS, and Aubrey A. Almeida, MBBS, Brisbane, Australia
}

From the Department of Cardiac Surgery, The Prince Charles Hospital, Brisbane, Australia.

Received for publication Feb. 18, 1997; accepted for publication May 28, 1997.

Address for reprints: Robert Tam, FRACS, Department of Cardiac Surgery, The Prince Charles Hospital, Rode Rd., Chermside, Brisbane, Australia, Q 4032.

J Thorac Cardiovasc Surg 1997;114:682-3

Copyright (C) 1997 by Mosby-Year Book, Inc.

$0022-5223 / 97 \$ 5.00+0 \quad \mathbf{1 2 / 5 4 / 8 3 5 6 2}$
A full resternotomy has been the standard approach for reoperation on the aortic valve. This carries a risk of right ventricular injury, which has a significant morbidity and mortality. ${ }^{1}$ Approaches to minimally invasive primary aortic valve replacement have been described. ${ }^{2,3}$ Our approach is to perform primary aortic valve surgery via a minimally invasive upper hemisternotomy without horizontal transection of the sternum. We describe a case of minimally invasive redo aortic valve replacement in which this approach was used.

A 69-year-old man had heart failure as a result of aortic valve xenograft degeneration. He had aortic valve replace- 
ment with a bioprosthesis 18 years previously for aortic valve bacterial endocarditis. He had had increasing dyspnea on exertion for 12 months before being admitted to our hospital.

Transesophageal echocardiography showed severe aortic xenograft regurgitation with leaflet prolapse. The ejection fraction was $55 \%$, with an end-diastolic dimension of $66 \mathrm{~mm}$. There was no evidence of prosthetic valve endocarditis. Coronary angiography showed wall disease in the left anterior descending artery.

An upper median hemisternotomy was performed with an oscillating saw. The sternum was divided from the notch to the fourth intercostal space. The sternum was not transected at the distal end (Fig. 1). A small retractor was placed to expose the ascending aorta and the right atrial appendage. Adhesions were freed and excessive thymic fat was excised to improve exposure. A tape was then placed around the aorta, and it was cannulated proximal to the brachiocephalic trunk. Because of the small incision and the previous use of the right atrial appendage for cannulation, repeat cannulation by means of this approach was not possible. The right femoral vein was then exposed and cannulated with a 27F-gauge venous cannula. Cardiopulmonary bypass was established and full flow was achieved by connecting the venous cannula to the roller pump. The patient was cooled to $30^{\circ} \mathrm{C}$. The aorta was crossclamped after the heart was drained by continuing venous suction while temporarily ceasing arterial inflow. This prevented left ventricular distention before cardioplegic arrest, because the ventricle could not be vented at this stage.

An oblique aortotomy extending to the noncoronary sinus was performed. Cold crystalloid cardioplegic solution was infused directly into both coronary ostia. The left ventricle was then vented through the aortic valve. The bioprosthesis was explanted and a size 21 CarpentierEdwards Perimount xenograft (Baxter Healthcare Corp., Edwards Division, Santa Ana, Calif.) was implanted with interrupted 2-0 Ethibond sutures (Ethicon, Inc., Somerville, N.J.). The vent was removed as the aortotomy was closed and the heart was deaired through an aortic root vent. The heart was reperfused and sinus rhythm returned. Cardiopulmonary bypass was discontinued. The femoral vein was repaired with a continuous 6-0 Prolene suture (Ethicon). A single chest drain was inserted through the right fifth intercostal space. The sternotomy was closed with three parasternal wires. The aortic crossclamp time was 59 minutes, with a total bypass time of 78 minutes.

The patient was extubated 7 hours after the operation and required no inotropic support. The blood loss was 400 $\mathrm{ml}$ and he was discharged from the intensive care ward the following day. Postoperative echocardiography showed normal aortic valve function. There were no embolic events. He had minimal wound discomfort and was discharged from the hospital on day 5.

The widely recognized complications of reoperative cardiac surgery are cardiac injury and increased bleeding. The major problem arises from adhesions between the right ventricle and the lower sternum. To avoid this problem, we modified our previous experience of aortic valve surgery by using an upper hemisternotomy. We have

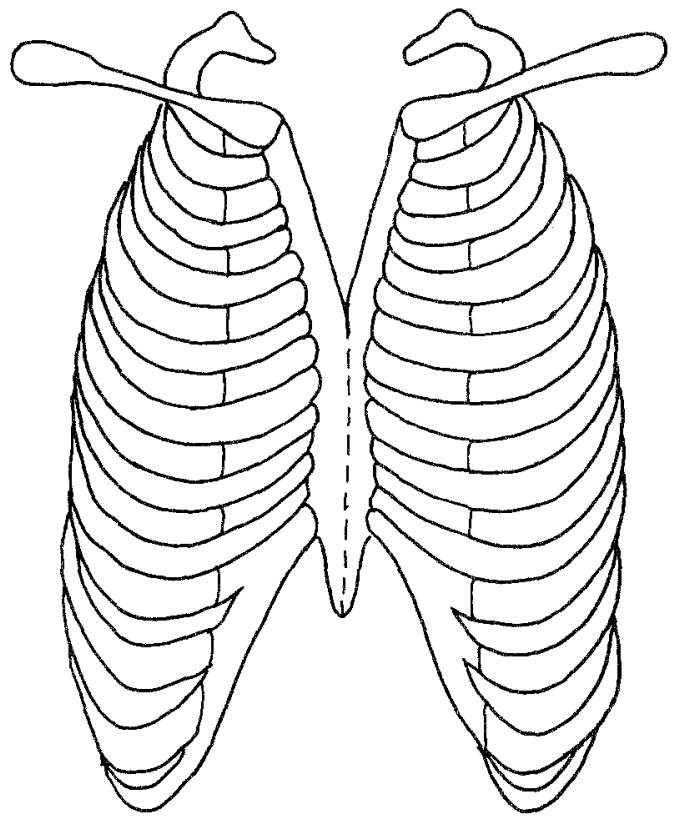

Fig. 1. Sketch demonstrating the upper hemisternotomy before closure. The dotted line indicates the extent of the previous sternotomy.

found that this approach is a safe alternative to full median sternotomy in primary aortic valve replacement. Some surgeons perform aortic valve surgery using an upper median sternotomy; however, their approach involves transecting the sternum. We have found this unnecessary to achieve adequate access. In addition, our approach does not risk injury to the internal thoracic artery, and the sternum is inherently stable.

In this case the exposure of the aorta was excellent. Owing to scarring of the right atrial appendage, femoral vein cannulation was required. Postoperative blood loss and wound morbidity were minimal. The patient was able to be mobilized and discharged early.

Enthusiasm for minimally invasive direct coronary artery bypass procedures continues to increase. The aim of less invasive procedures is to maintain the effectiveness of the operation, minimize cost, improve patient recovery, and shorten intensive care and hospital stay. These potential benefits encourage our application of minimally invasive aortic valve surgery. With further refinement of techniques, the minimally invasive approach can be applied to other cardiac surgical procedures.

\section{REFERENCES}

1. Dobell ARC, Jain AK. Catastrophic hemorrhage during redo sternotomy. Ann Thorac Surg 1984;37:273-8.

2. Cosgrove DM, Sabik JF. Minimally invasive approach for aortic valve operations. Ann Thorac Surg 1996;62:596-7.

3. Konertz W, Waldenberger F, Schmautzler M, Ritter J, Liau J. Minimal access valve surgery through superior partial sternotomy: a preliminary study. J Heart Valve Dis 1996;5:638-40. 